\title{
Diagrama de manejo de densidad y su aplicación a raleo en bosques de segundo crecimiento de Drimys winteri en el sur de Chile
}

\author{
Stand density management diagram and its thinning applications in Drimys winteri second-growth \\ forests in southern Chile
}

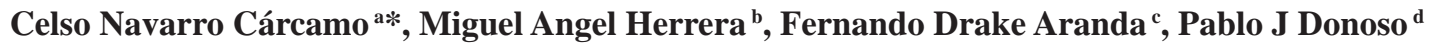 \\ *Autor de correspondencia: ${ }^{a}$ Universidad Católica de Temuco, Escuela de Ciencias Forestales, \\ Rudecindo Ortega 02950, Temuco, Chile, tel.: 56- 45- 205545, cnavarro@uct.cl \\ b Universidad de Córdoba, Córdoba, España. \\ ${ }^{c}$ Universidad de Concepción, Facultad de Ciencias Forestales, Concepción, Chile. \\ ${ }^{d}$ Universidad Austral de Chile, Facultad de Ciencias Forestales y Recursos Naturales, Valdivia, Chile.
}

\begin{abstract}
SUMMARY
Stand density management (DMD) diagrams are graphical models of uniform stands at different ages that reflect the relationship among size, density, competition, site occupancy and self thinning. DMDs allow planning of thinnings through the definition of a target stand and the upper and lower limits of site occupancy. In this study we developed a DMD for Drimys winteri second-growth forests in southern Chile by using 300 sampling units with at least $70 \%$ basal area of this species. The resulting DMD allowed the determination of the maximum density line, the volume and height isolines, and the evaluation of its use with information from permanent plots thinned 21 years ago. The results of the functions in the DMD present acceptable predictive power, and illustrate the usefulness and precision of this tool to plan silviculture activities. Results from applying the DMD suggest maintenance of site occupancy levels between the subutilization zone (30 \% relative density index (RDI)) and the imminent mortality zone (45 \% IDR), executing at least three thinnings to reach a target stand that will produce sawtimber and veneer at an age of at least 80 years for the study site. With this scheme the windfall risk is reduced and therefore so is the potential loss of production.
\end{abstract}

Key words: silvicultural management, relative density, canelo, self thinning.

\section{RESUMEN}

Los diagramas de manejo de la densidad (DMD) son modelos gráficos de la dinámica de rodales uniformes a diferentes edades que reflejan la relación entre tamaño, densidad, competencia, ocupación del sitio y autorraleo; y permiten la planificación de raleos mediante la definición de un rodal meta y los límites superior e inferior de ocupación del sitio. Este estudio desarrolló un DMD para renovales de Drimys winteri en el sur de Chile, utilizando 300 unidades muestrales con una participación mayor a 70 \% de área basal de D. winteri, que permitió determinar las líneas de tamaño máximo densidad, las isolíneas de volumen, isolíneas de altura, y evaluar su uso con datos de parcelas permanentes de raleo medidos durante 21 años. Los resultados de las funciones que conforman el DMD presentan aceptables capacidades predictivas, constatando la utilidad y precisión de esta herramienta para planificar intervenciones silviculturales. Los resultados de la aplicación del DMD sugieren mantener niveles de ocupación de sitio entre las zonas de subutilización (índice de densidad relativa (IDR) de $30 \%$ ) y la de inminente mortalidad por competencia (IDR de $45 \%$ ), realizando al menos tres raleos para alcanzar un rodal meta que genere productos aserrables y debobinables a una edad no menor a los 80 años para el sitio analizado. Con este esquema se reduce el riesgo de caída de árboles por viento y la pérdida de producción física del sitio.

Palabras clave: gestión silvicultural, densidad relativa, canelo, autorraleo.

\section{INTRODUCCIÓN}

El manejo de la densidad y su relación con estados de desarrollo y niveles de competencia, asociado a esquemas de manejo a nivel de rodal, requiere de herramientas que permitan cuantificar la densidad a dejar luego de una intervención silvicultural en relación a la ocupación máxima del sitio que puede hacer una especie. La densidad del rodal puede ser expresada en términos absolutos o relativos, en términos absolutos es determinada directamente desde un rodal dado sin referencia de algún otro rodal y se cuantifica normalmente con los parámetros de número de árboles por unidad de superficie, área basal y volumen de madera; en tanto, en términos relativos se basa sobre una densidad estándar seleccionada (Avery y Burkhart 1994). Estudiar la densidad y su vinculación con el desarrollo del rodal futuro es posible mediante el análisis de las relaciones de tamaño-densidad. Estas relaciones son básicas para determinar los índices de densidad del rodal, los cuales son utilizados para caracterizar estados de desarrollo como los de crecimiento libre, crecimiento con competencia y mortalidad debido a la competencia (Penner et al. 2002). La 
más conocida de las relaciones de tamaño-densidad es la ley de autorraleo, que fue definida separadamente por Reineke (1933) y Yoda et al (1963), la que describe una relación entre el tamaño y la densidad en rodales coetáneos, monoespecíficos, no intervenidos y en crecimiento activo: el primero determinó la denominada regla de la densidad del rodal que relaciona el número de árboles por hectárea con el diámetro medio cuadrático mediante una función lineal a escala logarítmica con una pendiente universal de -1,605; y, por su parte Yoda et al. (1963) determinaron la regla de autorraleo del $-3 / 2$, iniciando la más prominente y controversial discusión respecto de las escalas de las reglas (Pretzsch 2009).

Estas relaciones han sido utilizadas por diversos autores en el desarrollo de modelos matemáticos para describir el desarrollo temporal de rodales de distintas especies (Somers y Farrar 1991), siendo comúnmente expresadas a través de los siguientes modelos matemáticos [1] al [4]:

$\ln (\mathrm{N})=\alpha-1,605 \ln (\mathrm{DMC})($ Reineke 1933).

$\ln (\mathrm{VM})=\alpha-1,5 \ln (\mathrm{N})$ (Drew y Flewelling 1977).

$\ln (\mathrm{W})=\alpha-0,5 \ln (\mathrm{N})($ Zeide 1987).

$\ln (\mathrm{DMC})=\alpha-0,625 \ln (\mathrm{N})($ Jack y Long 1996).

Donde VM: volumen medio, W: total de biomasa o total de volumen, DMC: diámetro medio cuadrático, $\alpha$ : constante en la ecuación, N: número de árboles por hectárea, ln: logaritmo natural.

Una herramienta de manejo forestal desarrollada a partir de las relaciones tamaño densidad son los denominados diagramas de manejo de la densidad, que son esencialmente simples modelos promedio del rodal que caracterizan gráficamente rendimiento, densidad y mortalidad en varios estados de desarrollo (Newton y Weetman 1994, Shaw et al. 2007, Gezan et al. 2007, Vacchiano et al. 2008, Valbuena et al. 2008, Castedo et al. 2009). Los diagramas de manejo de la densidad constituyen una herramienta valiosa para la planeación de regímenes de tratamientos silviculturales que garanticen una óptima ocupación del sitio, orientando las acciones silviculturales para posicionar los rodales en valores de densidad o niveles de competencia de acuerdo con los objetivos de producción propuestos (Shaw y Long 2007).

Según Farden (2002), los diagramas de manejo de la densidad pueden ser agrupados de la siguiente forma: a) los más simples contienen las líneas de límite básicas que describen la zona de autorraleo y, posiblemente, la línea de cierre de copas y son una guía básica para el raleo de rodales o densidades de establecimiento; b) un segundo nivel de complejidad es alcanzado, agregando sistemas de isolíneas que representan la altura dominante y volumen o diámetro (dependiendo del formato), y son los de mayor uso hasta la fecha; c) un tercer nivel de complejidad se alcanza con la incorporación de curvas de mortalidad que agregan considerable precisión y exactitud a las predicciones; y d) menos comunes y menos desarrolladas son los que incorporan ejes adicionales, como combinaciones de especies y de calidad de sitio.

Nyland (2002) señala que las primeras guías de manejo de densidad no reconocen el efecto de la composición de especies per se, éstas se aplicaron a tipos de comunidades de bosques simples para una región específica o para rodales puros. Más tarde las investigaciones demostraron diferencias en la razón área-árbol entre grupos separados de especies y mostraron los beneficios de ajustar la densidad relativa, reflejando la composición de especies, para lo cual se han descrito tres opciones: a) presentar curvas diferentes para cada tipo de comunidad forestal particular o específica; b) presentar curvas de tamaño máximo-densidad para diferentes grupos de composición de especies o tipo de bosque y c) utilizar factores de conversión con base en el área basal por especie y la clase de tamaño, lo que produce una estimación de la densidad relativa que refleja la composición de especie en cada rodal por separado.

La utilidad de incorporar las isolíneas de altura y volumen permite tener una estimación del estado cuantitativo del rodal, y realizar una mejor proyección de las intervenciones propuestas. Al combinar los diagramas con funciones de altura dominante-edad es factible estimar el tiempo que tomará llegar a un estado de desarrollo para los esquemas de manejo proyectados y diferenciar tipos de raleos (Vacchiano et al. 2008, Castedo et al. 2009). Además, Farden (2002) plantea que las formas de las isolíneas de altura y volumen presentadas en escala logarítmica son poco afectadas por la densidad. Ello indica que la construcción de curvas más apropiadas para un mejor entendimiento de los usuarios de estas relaciones es un desafío que se debe afrontar, fundamentalmente en las relaciones entre densidad y varios parámetros alométricos. Sin embargo, si se asume que la altura dominante es una función de la edad y que es aproximadamente igual para rodales de edades similares, creciendo en lugares con aproximadamente la misma calidad de sitio, entonces es posible representar el componente temporal en dichos diagramas.

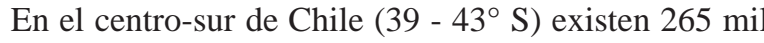
hectáreas de bosques de Drimys winteri Forst. (INFOR 2004), principalmente en condición de bosques secundarios. Debido a la importancia de estas formaciones, recientemente se han desarrollado estudios sobre silvicultura y ecología que incluyen resultados de largo plazo de tratamientos de raleos (Reyes et al. 2009) y evaluación de relaciones tamaño-densidad con variaciones de sitio (Donoso et al. 2007). Este último estudio determinó que dichas relaciones eran independientes del sitio, lo que sienta las bases para el desarrollo de un diagrama de manejo de la densidad para bosques secundarios de esta especie. Los diagramas de manejo de la densidad, aunque no reemplazan a un simulador, son prácticos y fáciles de utilizar por los silvicultores (Valbuena et al. 2008, Castedo 2009) y de 
gran aporte potencial a la gestión de los bosques naturales.

En la práctica silvicultural, el principal componente de la estructura cuantitativa del bosque que se modifica es la densidad, la que, expresada en términos relativos, permite relacionarla con estados de desarrollo y niveles de competencia. Al respecto, Drew y Flewelling (1979) estimaron para Pseudotsuga menziessi (Mirb.) Franco la línea de inminente mortalidad con una densidad relativa de 0,55 , la de inicio de la máxima producción del rodal con 0,40 y la línea de cierre de copas con 0,15. Newton (1997) utilizó índices de densidad relativa para Picea mariana (Mill.) BSP de 0,13 para la línea de cierre de copas y 0,5 en la línea de mortalidad inminente por competencia. En Chile, Gezan et al. (2007) construyeron un diagrama de manejo de la densidad para Nothofagus spp., sugiriendo considerar índices de densidad de rodal de $60 \%$ para la línea de mortalidad inminente por competencia, $30 \%$ en la línea del límite inferior de ocupación del sitio y $20 \%$ para la línea de cierre de copas.

Se plantea como hipótesis, que es factible desarrollar un diagrama de manejo de la densidad de segundo nivel de complejidad para renovales de $D$. winteri, fundamentado en los marcos teóricos del principio de autorraleo y la experiencia de su elaboración en otras especies a nivel mundial, con errores aceptables para la planificación y control de tecnologías silviculturales a base de niveles de ocupación de sitio; evitando así la pérdida de producción física del sitio y reduciendo el riesgo de caída de árboles por viento para un bosque objetivo. Este estudio tiene como objetivos: a) construir un diagrama de manejo de la densidad para bosques de segundo crecimiento de $D$. winteri en el sur de Chile y, b) evaluar el desempeño de tal diagrama en la planificación y evaluación de raleos. Estos bosques están dominados por $D$. winteri, se definen como coetáneos, en su gran mayoría no presentan intervenciones y se encuentran en estados de desarrollo de monte bravo, latizal y fustal delgado; lo cual permite encontrar en gran parte de los renovales condiciones donde se produce mortalidad natural por competencia.

\section{MÉTODOS}

Área de estudio. El estudio abarcó información de bosques secundarios de $D$. winteri entre los paralelos $40^{\circ}$ y $43^{\circ} \mathrm{S}$ y entre los meridianos $73^{\circ}$ y $74^{\circ} \mathrm{O}$, en altitudes de 50 a 600 m s.n.m. y pendientes menores a 20 \%. La región presenta un clima templado lluvioso, con temperaturas mínimas en julio de $-2{ }^{\circ} \mathrm{C}$ y máximas en enero de $30^{\circ} \mathrm{C}$ (Di Castri y Hajek 1976). La precipitación media anual varía entre 1.500 y $4.000 \mathrm{~mm}$ (Di Castri y Hajek 1976). Los suelos donde se establecen estos bosques secundarios de $D$. winteri son delgados (30 a $80 \mathrm{~cm}$ ), de mal drenaje, altamente ácidos y tienen una capa superficial de humus ácido que muestra un moderado grado de lixiviación de materia orgánica dentro del sustrato mineral (Donoso et al. 1985, 2006). Es posible encontrar áreas con suelos bien drenados a imperfectamente drenados, originados de cenizas volcánicas; sin embargo, se encuentran en distinto grado de empobrecimiento por el manejo deficiente y uso intensivo (Navarro et al.1999).

Fuente de información. Los datos utilizados para la construcción del diagrama de manejo de la densidad provienen de parcelas permanentes y otras temporales establecidas entre los años 1985 y 2007. Las unidades muestrales son rectangulares con superficies entre 500 y $1.000 \mathrm{~m}^{2}$, dependiendo del estado de desarrollo del bosque. En cada unidad muestral se registraron las coordenadas UTM, la fuente del inventario, año de medición, comuna, altitud, exposición, pendiente, posición fisiográfica, estado de desarrollo (monte bravo, latizal y fustal delgado), tipo de intervención en caso de que hubiera y cobertura de copa. A nivel de árbol se registró la especie, diámetro a la altura de 1,3 metros desde el suelo (DAP), sanidad, forma, dominancia y, a una muestra de árboles, se le registró la altura total, la altura comienzo de copa y el área de copa. La base de datos generada fue de 300 unidades muestrales en que predomina $D$. winteri con al menos $70 \%$ de participación en términos de área basal y volumen. Del total de unidades muestrales usadas, 177 no registraban intervención y 123 habían tenido algún grado de intervención.

Para cada unidad muestral se determinaron los parámetros dasométricos: densidad de rodal, diámetro medio cuadrático, área basal, volumen y la altura dominante a partir del promedio de los 100 árboles de mayor diámetro por hectárea (Penner et al. 2002, Long y Shaw 2005, Shaw y Long 2007, Vacchiano 2008, Castedo et al. 2009). Además se incluyeron variables dasométricas medidas o estimadas necesarias para la construcción del diagrama de manejo de la densidad. También, para cada parcela, se determinó el índice de densidad relativa (Curtis 1970), el cual se define como la razón entre el número de árboles por unidad de superficie de un rodal (Na) y el máximo número (Nmax) de árboles que podría alcanzar para el mismo tamaño promedio, ya sea este expresado en diámetro medio cuadrático o volumen medio de árbol. El índice de densidad relativa se obtuvo a partir de la curva que relaciona el tamaño medio máximo con la densidad [5]:

\section{$\mathrm{IDR}=\mathrm{Nad} / \mathrm{Nmaxd}$}

Donde, IDR: índice de densidad de relativa, Nad: número de árboles por hectárea para un tamaño dado "d" de diámetro medio cuadrático (DMC), Nmaxd: máximo número de árboles estimado con la función de tamaño medio máximo densidad para el tamaño "d".

De los datos utilizados para la construcción del diagrama de manejo de la densidad, destacó la amplitud de las variables dasométricas que en términos de densidad fue de 333 a 10.350 árboles ha-1 $, 6,8$ a 36,2 cm para el diámetro medio cuadrático, 52 a $874 \mathrm{~m}^{3}$ ha $^{-1}$ en volumen y de 10 a $25 \mathrm{~m}$ en altura (cuadro 1). 
Cuadro 1. Estadística descriptiva de los datos usados para la construcción del diagrama de manejo de la densidad. Descriptive statistics of the data used for the construction of the density management diagram (DMD).

\begin{tabular}{lrrrrrrrrrrrr}
\hline \multirow{2}{*}{ Parámetro } & \multicolumn{4}{c}{ Todos los rodales } & \multicolumn{1}{c}{ Rodales sin manejo } \\
\cline { 2 - 13 } & $\mathrm{N}$ & $\mathrm{AB}$ & $\mathrm{V}$ & DMC & Hm & IDR & $\mathrm{N}$ & AB & $\mathrm{V}$ & DMC & Hm & IDR \\
\hline Mínimo & 333 & 8,8 & 52,1 & 6,8 & 10,0 & 0,09 & 420 & 19,6 & 125,7 & 6,8 & 10,0 & 0,19 \\
Máximo & 10.350 & 102,8 & 874,4 & 36,2 & 25,5 & 1,10 & 10.350 & 102,8 & 874,4 & 36,2 & 25,5 & 1,10 \\
Media & 3.409 & 53,1 & 377,9 & 16,1 & 17,0 & 0,56 & 4.173 & 63,3 & 461,8 & 15,1 & 17,4 & 0,66 \\
CV (\%) & 67 & 41,9 & 49,8 & 31,5 & 13,6 & 42,39 & 47 & 29,8 & 36,8 & 32,3 & 15,5 & 31,55 \\
\hline
\end{tabular}

$\mathrm{N}$ : número de árboles por hectárea, $\mathrm{AB}$ : área basal $\left(\mathrm{m}^{2} \mathrm{ha}^{-1}\right)$, V: volumen total $\left(\mathrm{m}^{3} \mathrm{ha}^{-1}\right)$, DMC: diámetro medio cuadrático (cm), Hm: altura máxima (m), IDR: índice de densidad relativa, CV (\%): coeficiente de variación.

Construcción del diagrama de manejo de la densidad. Para construir el diagrama de manejo de la densidad y evaluar las relaciones entre las funciones que lo integran, se utilizó la base de datos que se generó, seleccionando las parcelas que cumplían con el criterio de bosques puros de D. winteri (cuadro1).

Línea de máximo tamaño-densidad. El tamaño máximo promedio a nivel de árbol para cualquier densidad se obtuvo a través de la relación conocida como la ley del autorraleo (Drew y Flewelling 1979, Solomon y Zhang 2002, Donoso et al. 2007), que fue propuesta por Tadaki y Shidei (1959) y generalizada por Yoda et al. (1963). Cuando el logaritmo de la masa promedio es graficado versus el logaritmo de la densidad de las plantas, los puntos forman una línea recta llamada "línea de autorraleo" de la forma como se indica en la ecuación [6]:

$\ln W=k-3 / 2 \ln ($ árboles ha-1)

Donde $\ln \mathrm{W}$ : logaritmo natural del tamaño promedio del árbol, ln (árboles ha-1): logaritmo natural de la densidad, $k$ : constante que varia con la especie.

Para este estudio se ajustó la ecuación propuesta por Jack y Long (1996), que consideró el logaritmo natural del diámetro medio cuadrático como la variable de tamaño medio, priorizando la predicción y utilidad práctica por sobre la lógica explicativa, quedando la función [7] de tamaño versus densidad, de la siguiente forma:

$\ln \mathrm{DMC}=\alpha+\beta \ln \mathrm{N}$

Donde ln DMC = logaritmo natural del diámetro medio cuadrático, ln $\mathrm{N}$ = logaritmo natural de la densidad (árboles ha $\left.^{-1}\right) ; \alpha, \beta=$ parámetros del modelo.

La determinación de la línea máxima tamaño-densidad se realizó a través de análisis de regresión mediante el método de los mínimos cuadrados ordinarios corregidos (Gujarati 2003, Zhang et al. 2005), utilizándose las parcelas sin intervención. En una primera etapa, al ajustar el modelo se obtiene una línea de autorraleo considerada promedio, que representa todos los datos y permite la existencia de puntos sobre la línea (Solomon y Zhang 2002). Luego, para obtener el límite máximo de la línea de autorraleo, se identificaron las tres unidades muestrales que presentan el máximo residual y se obtiene el valor promedio residual máximo (max ei), cifra que se suma al parámetro interceptor $\alpha$, generando una línea paralela que representa la máxima relación tamaño densidad (Zhang et al. 2005), procediendo de la siguiente manera [8]:

$\mathrm{MRTD}=\alpha+\max$ ei

Donde MRTD: máxima relación tamaño densidad, max ei: máximo residual, $\alpha$ : parámetro interceptor.

Isolíneas de volumen. Las isolíneas de volumen se ajustaron agregando las unidades muestrales que presentaban intervenciones, de modo de contar con diferentes niveles de competencia y ocupación del sitio. La estimación del volumen se realizó mediante el uso de funciones generales a nivel de árbol, utilizando el DAP y altura total como variables predictoras (Navarro et al. 1999). Se evaluaron modelos probados con buenos resultados por diversos autores (Penner et al. 2002, Newton 1997, Shaw y Long 2007). El modelo utilizado por Vacchiano (2008) fue el que presentó un mejor comportamiento, tanto en el ajuste como en sus capacidades predictivas. Las isolíneas de volumen se generaron expresando el volumen total del rodal en función del diámetro medio cuadrático y la densidad, mediante la siguiente ecuación [9]:

$V=b_{1} N\left(D M C-b_{2}\right)^{b_{3}}$

Donde V: volumen del rodal en $\mathrm{m}^{3} \mathrm{ha}^{-1}$, $\mathrm{N}$ : densidad del rodal (árboles ha-1), DMC: diámetro medio cuadrático $(\mathrm{cm})$; $b_{1}, b_{2}, b_{3}$ : parámetros del modelo.

Esta ecuación modela muy bien la relación entre el volumen medio del árbol y el diámetro medio cuadrático, produciendo curvas de volumen ligeramente cóncavas cuando es trazado sobre un diagrama de manejo de la densidad. 
Isolíneas de altura dominante. Las parcelas usadas para ajustar las isolíneas de volumen también se utilizaron para ajustar las isolíneas de altura dominante. Las isolíneas de altura dominante se generaron con un modelo no lineal que relaciona la altura dominante con el diámetro medio cuadrático y la densidad [10]:

$$
H_{100}=D M C /\left(b_{1}-b_{2} \ln (N)\right)
$$

Donde $\mathrm{H}_{100}$ : altura media de los cien árboles dominante (m), N: densidad del rodal (árboles ha ${ }^{-1}$ ), DMC: diámetro medio cuadrático $(\mathrm{cm}) ; b_{1}, b_{2}$ : parámetros del modelo.

En este modelo se fuerza el signo negativo del coeficiente $b_{2}$, de tal manera que explique la influencia inversa de la densidad del rodal sobre el diámetro de árbol. Previamente se probaron otros modelos que no arrojaron mejores resultados en términos de la bondad del ajuste y capacidades predictivas (Drew y Flewelling 1979, Penner et al. 2002, Long y Shaw 2005, Castedo et al. 2009).

Los modelos se evaluaron a través del coeficiente de determinación $\left(\mathrm{R}^{2}\right)$, el error estándar de la estimación (Syx), la diferencia agregada (DIFA) y la raíz del error medio cuadrático (REMC) [11], [12]:

$R E M C \%=\frac{\sqrt{\frac{\sum_{i=1}^{n}\left(Y_{i}-\hat{Y}_{i}\right)^{2}}{n}}}{\bar{Y}} \bullet 100$

$$
\text { DIFA\% }=\frac{\frac{\sum_{i=1}^{n}\left(Y_{i}-\hat{Y}_{i}\right)}{n}}{\bar{Y}} \bullet 100
$$

Donde $\hat{Y}_{\mathrm{i}}$ valor estimado, $\mathrm{Y}_{\mathrm{i}}$ valor observado, $\mathrm{n}$ : número de observaciones, $\bar{Y}$ : media observados.

Aplicación del diagrama de manejo de la densidad. Para efectos de evaluar el uso del diagrama se trazaron los parámetros dasométricos de intervenciones de raleo de un ensayo con cuatro tratamientos correspondientes a diferentes intensidades, que fue establecido el año 1985 en la zona de la Cordillera de la Costa de Valdivia, Chile $\left(40^{\circ} 07^{\prime} \mathrm{S}, 73^{\circ}\right.$ 23' O), y que ha sido medido periódicamente cinco veces hasta el año 2007. En este estudio se aplicaron intensidades de raleo moderado (raleo a $2 \mathrm{~m}$ ), fuerte (raleo a $3 \mathrm{~m}$ ) y severo (raleo a $4 \mathrm{~m}$ ), reduciendo las densidades relativas promedio de 0,60 de los bosques sin manejo a cifras de $0,37,0,21$ y 0,12 , respectivamente. El tratamiento de raleo moderado fue intervenido en una segunda oportunidad después de 11 años de aplicada la primera intervención, cuando el bosque alcanzó un índice de densidad relativa promedio de 0,49 y presentaba signos de mortalidad por competencia, reduciendo la densidad relativa a una cifra de 0,35 de IDR. Más detalles sobre este ensayo puede encontrarse en Reyes et al. (2009).

Finalmente se ilustró el uso del diagrama de manejo de la densidad mediante la planificación de esquemas de manejo, para lo cual se definió un rodal meta, niveles de competencia y ocupación de sitio.

\section{RESULTADOS}

Diagrama de manejo de la densidad. La densidad relativa de las unidades muestrales usadas en este estudio varió entre 0,07 y 1,0. El $32 \%$ de las unidades muestrales presentó una densidad relativa sobre 0,55 ; alrededor de un $25 \%$ se ubicó entre 0,40 a 0,55 y un $43 \%$ presentó de 0,15 a 0,40 (cuadro 2). Al considerar los bosques sin intervención aparente, el $73 \%$ presentó un valor mayor o igual a 0,40 y un $53 \%$ sobre 0,55 , lo que indicó el alto nivel de competencia y mortalidad que está ocurriendo en estos bosques (cuadro 2). La relación entre el diámetro medio cuadrático y la densidad de las parcelas sin intervención de la figura 1, expresa la trayectoria del autorraleo y los elevados niveles de ocupación de sitio de estos bosques.

En el caso del modelo que relaciona tamaño medio y la densidad se obtuvo un $\mathrm{R}^{2}$ de $0,9146(P<0,001)$; para el modelo de isolíneas de volumen se observa una correlación fuerte y positiva entre el volumen total del rodal con la densidad y el diámetro medio cuadrático, con un $\mathrm{R}^{2}$ de $0,832(P<0,001)$; y para el caso del modelo de isolíneas de altura que relaciona el diámetro medio cuadrático con la altura dominante promedio y la densidad, la correlación está dada por un $\mathrm{R}^{2}$ de $0,776(P<0,001)$. En el caso de la bondad de predicción se observó que los tres modelos son insesgados con DIFAS menores a $2 \%$, y los errores de estimación (REMC\%), para los modelos a y b son 6,28 \%

Cuadro 2. Distribución de frecuencias de la densidad relativa para todos los rodales, para rodales sin manejo y para rodales intervenidos.

Frequency distribution of the relative density for all stands, for stands without management and for stands with management.

\begin{tabular}{lccc}
\hline $\begin{array}{c}\text { Índice de } \\
\text { densidad relativa }\end{array}$ & $\begin{array}{c}\text { Total } \\
(\%)\end{array}$ & $\begin{array}{c}\text { No intervenido } \\
(\%)\end{array}$ & $\begin{array}{c}\text { Intervenido } \\
(\%)\end{array}$ \\
\hline$<0,15$ & 3,8 & 0,7 & 3,1 \\
$0,15-0,30$ & 20,0 & 5,9 & 14,1 \\
$0,30-0,40$ & 18,9 & 9,0 & 9,9 \\
$0,40-0,55$ & 24,8 & 16,2 & 8,6 \\
$0,55-0,70$ & 18,4 & 14,8 & 3,5 \\
$0,70-0,85$ & 10,7 & 10,3 & 0,3 \\
$0,85-1,00$ & 2,8 & 2,1 & 0,7 \\
1,0 & 0,7 & 0,7 & 0,0 \\
\hline Total & 100,0 & 59,7 & 40,3 \\
\hline
\end{tabular}




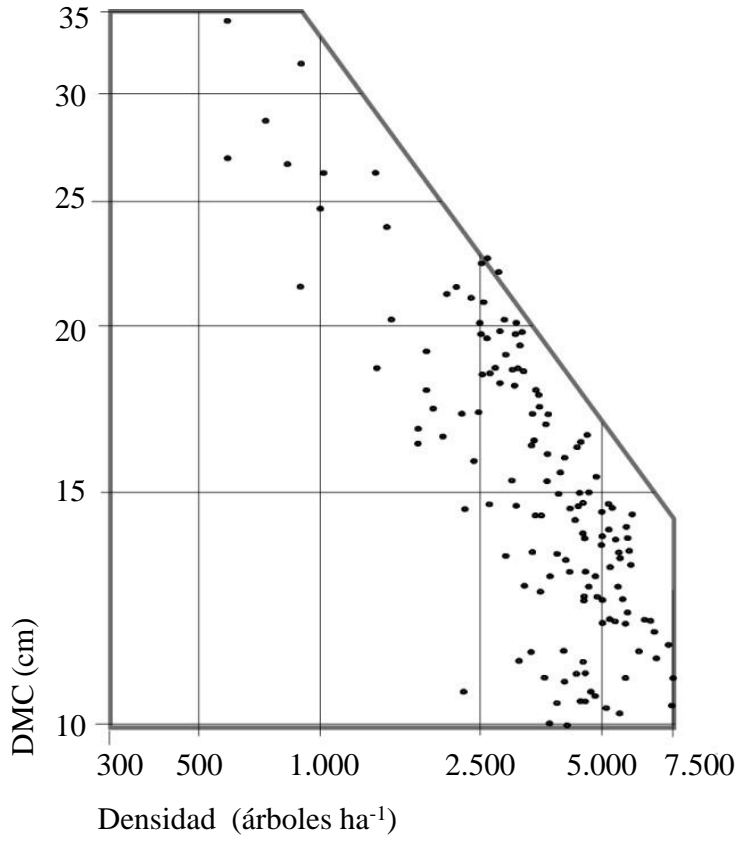

Figura 1. Relación del diámetro medio cuadrático (DMC) y densidad (árboles ha-1) de las parcelas sin intervención usadas en el estudio y línea de autorraleo.

Relationship between the average quadratic diameter (DMC) and density (trees ha ${ }^{-1}$ ) without intervention plots used in the study and line of self-thinning.

y $11,7 \%$, respectivamente; el modelo c tuvo una menor capacidad predictiva con una cifra de 16,2 \% (cuadro 3).

La función de tamaño máximo-densidad determinada según el modelo (a) del cuadro 3 y un intercepto corregido a partir del parámetro $b_{1}$ del modelo ajustado (a), cuyo valor es 6,68864, define la línea de autorraleo que se expresa de la siguiente forma [13]:

$\ln \mathrm{DMC}=6,68864-0,463137 \ln \mathrm{N}$

En el diagrama es posible identificar las líneas de densidad relativa, las isolíneas de volumen y las isolíneas de altura media dominante (figura 2). Las líneas de densidad relativa comienzan con la de autorraleo o máxima relación tamaño densidad y corresponde al límite derecho del diagrama de manejo de la densidad. A partir de esta y en forma paralela se trazaron otras cuatro líneas de densidad relativa, sobre la base de la evaluación de las parcelas permanentes y los estudios desarrollados por Venegas (1999) y Navarro et al. (1999): una primera que marca el inicio de la mortalidad, con una densidad relativa de 0,55 ; una segunda línea de una densidad relativa de 0,40 que define las condiciones donde los árboles ocupan totalmente el

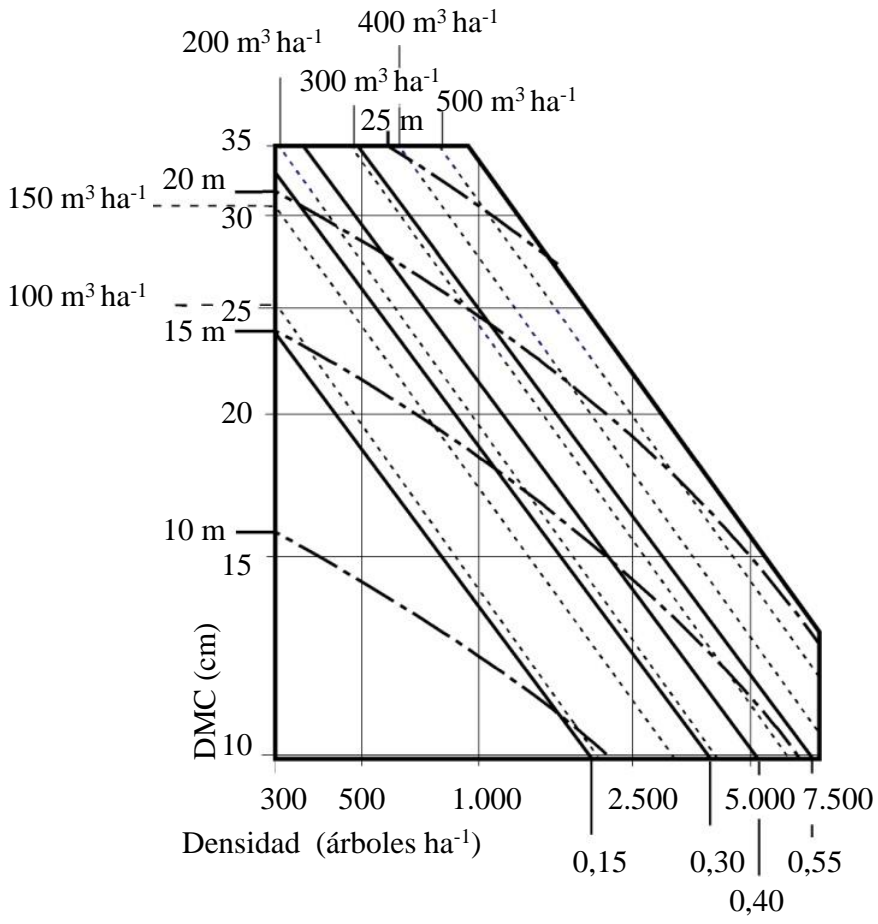

$\begin{array}{ll} & \text { IDR } \\ \ldots- & \text { Isolíneas de altura (m) } \\ \text {-.-- } & \text { Isolíneas de volumen }\left(\mathrm{m}^{3} \mathrm{ha}^{-1}\right)\end{array}$

Figura 2. Diagrama de manejo de la densidad. Density management diagram (DMD).

sitio y el rodal es altamente productivo; una tercera línea de densidad relativa de 0,30 que define el límite inferior de raleo para objetivos de producción de trozas aserrables o debobinables; y finalmente una cuarta línea de densidad relativa de 0,15 que indica el cierre de copas y el límite superior de la zona de crecimiento libre. Estos resultados son coincidentes con las determinadas por Drew y Flewelling (1979) para P. menziessi.

El uso del diagrama de manejo de la densidad es aplicable para renovales de $D$. winteri que presentan diámetro medio cuadrático que van desde los 10 hasta los $35 \mathrm{~cm}$ y densidades entre 350 a 7.500 árboles ha $^{-1}$, dado que en este universo se encuentran más del $98 \%$ de las unidades muestrales usadas en el estudio.

Las isolíneas de volumen se representaron por seis líneas que estiman volúmenes de 100,150,200,300, 400 y $500 \mathrm{~m}^{3} \mathrm{ha}^{-1}$ y por interpolación se obtuvieron los valores intermedios para los rodales, cuya estimación se realiza ubicando un rodal en el diagrama e interceptando su diámetro medio cuadrático y densidad. Para el caso de las isolíneas de altura dominante, éstas se representaron por cuatro líneas que indican alturas de 10, 15, 20 y 25 metros. 
Cuadro 3. Modelos de tamaño-densidad, de isolíneas de volumen e isolíneas de altura dominante. Models for size-density relationships of volume isolines and of dominant height isolines.

\begin{tabular}{ccccccc}
\hline Modelo & $\mathrm{b} 1$ & $\mathrm{~b} 2$ & $\mathrm{~b} 3$ & $\mathrm{R}^{2}$ & DIFA (\%) & REMC (\%) \\
\hline (a) $\ln d m c=\alpha+\beta \ln N$ & 6,621821 & $-0,463137$ & - & & & \\
Error estándar & 0,148642 & 0,018113 & - & 0,914 & $-0,625$ & $\pm 6,28$ \\
\hline $\begin{array}{c}\text { (b) } V=b_{1} N\left(D M C-b_{2}\right)^{b_{3}} \\
\text { Error estándar }\end{array}$ & 0,000290 & $-0,687828$ & 2,161730 & & & \\
\hline$H_{100}=D M C /\left(b_{1}-b_{2} \ln (N)\right)$ & 0,000213 & 1,429610 & 0,192090 & & & \\
$\quad$ Error estándar & 0,262214 & $-0,294920$ & - & & & $\pm 11,70$ \\
\hline
\end{tabular}

DIFA: diferencia agregada, REMC: raíz del error medio cuadrático.

Aplicación del diagrama de manejo de la densidad para definir tecnologías silvícolas en ensayo de raleo en la Cordillera de la Costa de Valdivia, Chile. La aplicación del diagrama de manejo de la densidad en el ensayo de raleo, determinó que el tratamientos de raleo fuerte después de 21 años desde la intervención presentó un índice de densidad relativa de 0,43 , ubicándose en la zona de inminente mortalidad por competencia. El raleo severo presentó un índice de densidad relativa de 0,32 , lo que indica una subutilización del sitio. Por su parte, el tratamiento testigo presentó un valor del índice sobre un 0,55 y se ubica en la zona de mortalidad por competencia, muy cerca de la línea del autorraleo (cuadro 4 y figura 3 ).

El raleo moderado después de 11 años de aplicada la primera intervención se ubicó en la zona de plena ocupación del sitio con un índice de densidad relativa de 0,49 , presentando árboles muertos por competencia. Se decidió en este caso realizar un segundo raleo que bajó el índice a 0,35 a la edad de 41 años, y luego de 11 años de transcurrido el segundo raleo el rodal alcanzó un valor de 0,40 , ubicándose en el comienzo de la zona de plena ocupación del sitio (cuadro 4).

Se determinaron crecimientos para el período de $11,7 \mathrm{~cm}$ (E1), 12,8 cm (E2), $15,8 \mathrm{~cm}$ (E3) y 4,3 cm (E4) y en términos de crecimiento anual periódico (CAP) se alcanzaron cifras de $0,55,0,61,0,75$ y $0,20 \mathrm{~cm}_{\text {año }}{ }^{-1}$, respectivamente. Esto se explica por una menor competencia y aumento significativo del área de copa en los árboles liberados, alcanzando un área de $11,85 \mathrm{~m}^{2}$ árbol ${ }^{-1}$ para el tratamiento del raleo severo, $6,14 \mathrm{~m}^{2}$ árbol-1 para el raleo fuerte, $5,53 \mathrm{~m}^{2}$ árbol ${ }^{-1}$ para el moderado y $4,60 \mathrm{~m}^{2}$ árbol ${ }^{-1}$ para los árboles dominantes del tratamiento testigo (cuadros 4 y 5). Estos resultados indicaron que a menor índice de densidad relativa residual se produjo un aumento del desarrollo de la copa de los árboles dejados en el raleo, y un mayor tamaño del árbol en términos de diámetro y volumen.

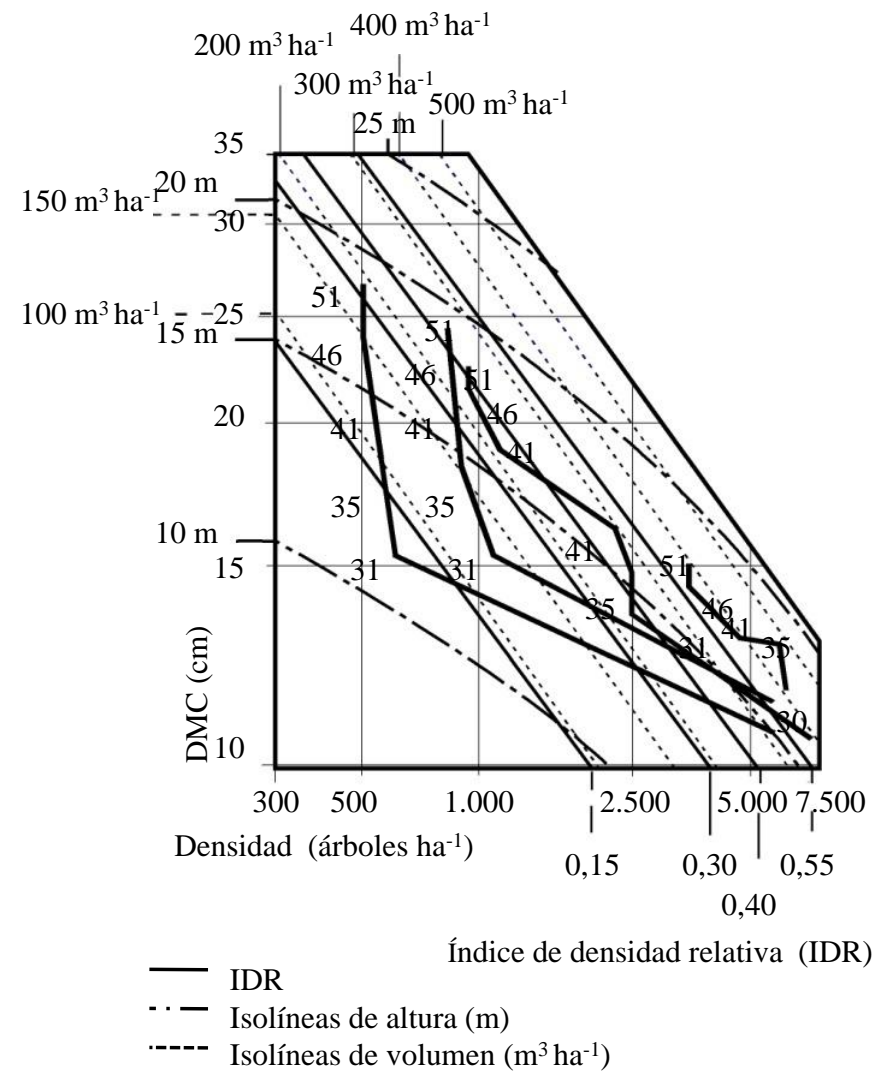

Figura 3. Trazado en el diagrama de manejo de la densidad (DMD) de los ensayos de raleo instalados en Hueicolla, provincia de Valdivia, y mediciones desde el año 1985 (30 años) hasta el año 2007 (51 años).

Drawn in trials (DMD) density management diagram of thinning installed in Hueicolla, province of Valdivia, and measurements since 1985 (30 years) until 2007 (51 years).

Planificar una trayectoria de raleos en un renoval de $D$. winteri de una edad promedio de 30 años, que inicialmente presentó un índice de densidad relativa de $0,60 \mathrm{y}$ reducirlo a cifras que van de 0,15 a 0,25 significó experi- 
Cuadro 4. Parámetros dasométricos de ensayo de raleo instalado en Hueicolla, provincia de Valdivia, para el período 1985 - 2007. Dasometric parameters of thinning experiments in secondary forest at Hueicolla, province of Valdivia, for the period 1985 to 2007.

\begin{tabular}{|c|c|c|c|c|c|c|c|}
\hline Tratamientos & Esquema de raleo & $\begin{array}{c}\text { Densidad } \\
(\text { árboles ha-1) }\end{array}$ & $\mathrm{DMC}(\mathrm{cm})$ & Año & $\begin{array}{l}\text { Edad } \\
\text { (años) }\end{array}$ & IDR & $\begin{array}{l}\text { Volumen bruto } \\
\qquad\left(\mathrm{m}^{3} \mathrm{ha}^{-1}\right)\end{array}$ \\
\hline \multirow{7}{*}{$\begin{array}{l}\text { Raleo moderado } \\
\text { (E1) }\end{array}$} & Estado inicial & 7.006 & 10,6 & 1985 & 30 & 0,61 & 395,8 \\
\hline & Raleo 1 & 2.494 & 13,6 & 1986 & 31 & 0,37 & 216,7 \\
\hline & Estado intermedio & 2.500 & 14,6 & 1990 & 35 & 0,44 & 254,6 \\
\hline & Estado intermedio & 2.228 & 16,2 & 1996 & 41 & 0,49 & 285,5 \\
\hline & Raleo 2 & 1.139 & 19,0 & 1996 & 41 & 0,35 & 189,3 \\
\hline & Estado intermedio & 928 & 21,4 & 2001 & 46 & 0,37 & 228,6 \\
\hline & Estado Intermedio & 928 & 22,3 & 2007 & 51 & 0,40 & 240,1 \\
\hline \multirow{6}{*}{$\begin{array}{l}\text { Raleo fuerte } \\
\quad \text { (E2) }\end{array}$} & Estado inicial & 5.678 & 11,4 & 1985 & 30 & 0,58 & 361,0 \\
\hline & Raleo 1 & 1.094 & 15,3 & 1986 & 31 & 0,21 & 125,2 \\
\hline & Estado intermedio & 916 & 18,0 & 1990 & 35 & 0,25 & 148,6 \\
\hline & Estado intermedio & 867 & 20,9 & 1996 & 41 & 0,33 & 194,5 \\
\hline & Estado intermedio & 828 & 23,4 & 2001 & 46 & 0,40 & 238,5 \\
\hline & Estado intermedio & 828 & 24,2 & 2007 & 51 & 0,43 & 251,3 \\
\hline \multirow{6}{*}{$\begin{array}{l}\text { Raleo Severo } \\
\text { (E3) }\end{array}$} & Estado inicial & 5.637 & 10,7 & 1985 & 30 & 0,50 & 309,1 \\
\hline & Raleo 1 & 611 & 15,3 & 1986 & 31 & 0,12 & 65,8 \\
\hline & Estado intermedio & 572 & 18,0 & 1990 & 35 & 0,16 & 90,7 \\
\hline & Estado intermedio & 534 & 20,9 & 1996 & 41 & 0,20 & 118,3 \\
\hline & Estado intermedio & 506 & 23,9 & 2001 & 46 & 0,26 & 151,1 \\
\hline & Estado intermedio & 506 & 26,5 & 2007 & 51 & 0,32 & 179,8 \\
\hline \multirow{5}{*}{$\begin{array}{l}\text { Sin manejo } \\
\quad \text { (E4) }\end{array}$} & Estado inicial & 6.174 & 11,7 & 1985 & 30 & 0,67 & 354,7 \\
\hline & Estado intermedio & 5.955 & 12,8 & 1990 & 35 & 0,78 & 404,8 \\
\hline & Estado intermedio & 4.583 & 13,0 & 1996 & 41 & 0,62 & 408,2 \\
\hline & Estado intermedio & 3.455 & 14,4 & 2001 & 46 & 0,59 & 423,8 \\
\hline & Estado intermedio & 3.483 & 15,0 & 2007 & 51 & 0,64 & 439,4 \\
\hline
\end{tabular}

DMC: diámetro medio cuadrático, IDR: índice de densidad relativa.

Cuadro 5. Índice de densidad relativa (IDR), mortalidad, área de copa promedio y crecimiento anual periódico (CAP) por tratamiento de raleo.

Relative density index (IDR), mortality, average crown area and periodic annual increment (CAP) for treatment of thinning.

\begin{tabular}{lcccc}
\hline Tratamiento & IDR & Mortalidad & $\begin{array}{c}\text { Área de } \\
\text { copa } \\
\left(\mathrm{m}^{2} \text { árbol }\right.\end{array}$ & $\begin{array}{c}\text { CAP } \\
(\mathrm{cm})\end{array}$ \\
\hline E1 (moderado) & 0,49 & Baja & 5,53 & 0,55 \\
E2 (fuerte) & 0,43 & No presenta & 6,14 & 0,61 \\
E3 (severo) & 0,32 & No presenta & 11,85 & 0,75 \\
E4 (testigo) & 0,64 & Alta & 4,60 & 0,20 \\
\hline
\end{tabular}

mentar caída de árboles por viento, si bien la copa se desarrolla y aumenta el diámetro a nivel de árbol la producción física del sitio es menor.

Planificación de intervenciones de raleo en renovales de D. winteri. Para la aplicación del diagrama de manejo de la densidad se definió un rodal meta cuyas características son un diámetro medio cuadrático de $35 \mathrm{~cm}$ y una densidad de 400 árboles cosecha por hectárea. Se estableció un límite superior de ocupación de sitio correspondiente a una densidad relativa de 0,45 y un límite inferior del rodal de 0,30 , que tuvo como objetivo evitar la mortalidad relacionada a la densidad y permitió mantener la copa viva con un buen vigor y evitar la caída por vientos. Las intervenciones o 
Cuadro 6. Esquemas de intensidad y periodicidad de raleos tardíos en base al uso de diagrama del manejo de la densidad para bosques secundarios de $D$. winteri (ES1: 3 raleos; ES2: 4 raleos).

Schemes of thinning intensity and periodicity based on the density management diagram (DMD) for secondary D. winteri forests (ES1: 3 thinnings; ES2: 4 thinnings).

\begin{tabular}{llcccc}
\hline Escenario & Esquema de raleo & IDR & Densidad (árboles ha $\left.{ }^{-1}\right)$ & DMC (cm) & Edad (años) \\
\hline \multirow{4}{*}{ ES1 } & Estado final & 0,46 & 400 & 35 & 75 \\
& Raleo 3 & 0,30 & 400 & 29 & 52 \\
& Estado intermedio & 0,45 & 900 & 24 & 52 \\
& Raleo 2 & 0,30 & 900 & 20 & 38 \\
& Estado intermedio & 0,45 & 2.000 & 16 & 38 \\
& Raleo 1 & 0,30 & 2.000 & 14 & 30 \\
Estado inicial & 0,62 & 6.500 & 11 & 30 \\
\hline \multirow{4}{*}{ ES2 } & Estado final & 0,46 & 400 & 35 & 75 \\
& Raleo 4 & 0,30 & 400 & 29 & 60 \\
& Estado intermedio & 0,45 & 700 & 27 & 60 \\
Raleo 3 & 0,30 & 700 & 23 & 45 \\
& Estado intermedio & 0,45 & 1.200 & 21 & 45 \\
Raleo 2 & 0,30 & 1.200 & 18 & 35 \\
& Estado intermedio & 0,45 & 2.500 & 15 & 35 \\
Raleo 1 & 0,40 & 2.500 & 14 & 30 \\
& Estado inicial & 0,62 & 6.500 & 11 & 30 \\
\hline
\end{tabular}

IDR: índice de densidad relativa; DMC: diámetro medio cuadrático.

tecnología silvícola para alcanzar el rodal meta consideran dos escenarios ES1 y ES2 (cuadro 6), que corresponden a los más factibles a implementar operacionalmente para renovales de productividad media de canelo. En el escenario 1 , se planificaron tres raleos, la intensidad del primer raleo contempló un $32 \%$ de reducción en el índice de densidad relativa (de 62 a $30 \%$ ), y la de los dos siguientes de $15 \%$ en cada caso; la periodicidad entre el primer y segundo raleo fue de 8 años, y entre el segundo y el tercer raleo de 14 años. En el escenario 2 se realizaron cuatro raleos, la intensidad del primer raleo contempló un 22 \% de reducción en el índice de densidad relativa y de los tres siguientes raleos un $15 \%$, la periodicidad entre el primer y segundo raleo fue de cinco años, entre el segundo y tercero 10 años, entre el tercero y cuarto 15 años y después de 10 años se realiza la corta final.

Las líneas de raleo trazadas en el diagrama de manejo de la densidad ilustraron la intervención, identificando segmentos inclinados que representan zonas de raleo y segmentos verticales que representan zonas de postraleo (figura 4). En el caso de las líneas verticales (post raleo), el rodal se caracteriza por la mantención de la densidad y aumento del diámetro medio cuadrático. En el caso de las líneas inclinadas, éstas representaron la extracción de individuos suprimidos, intermedios y codominantes (raleo por lo bajo grado C; Nyland 2002), donde la pendiente del

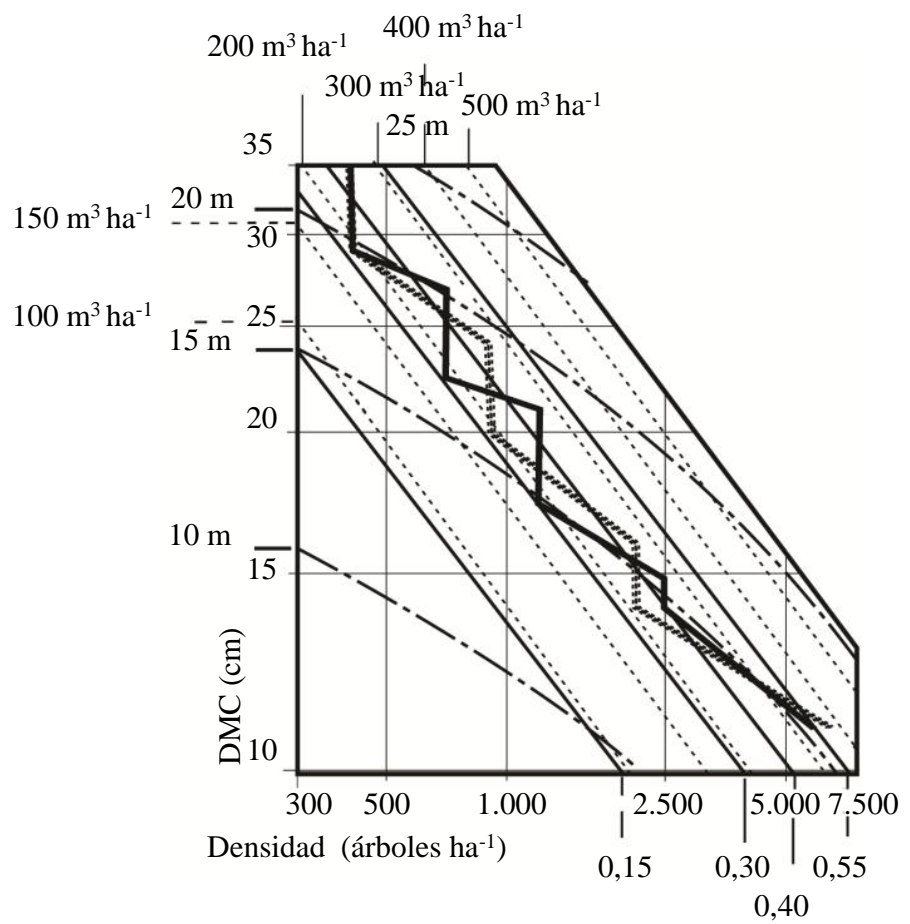

Índice de densidad relativa (IDR)
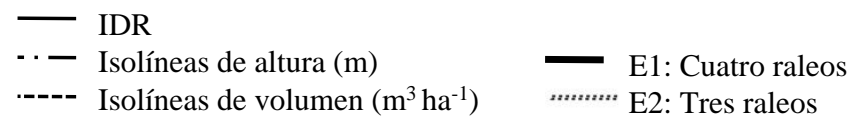

Figura 4. Propuesta de raleos tardíos con uso del diagrama de manejo de la densidad para renovales de $D$. winteri. Proposal of later thinning using DMD for $D$. winteri second- 
raleo no sigue estrictamente las isolíneas de alturas dominantes que es lo que ocurre al realizar un raleo estrictamente por lo bajo, suponiendo que el raleo no tiene ningún efecto sobre esta altura.

\section{DISCUSIÓN Y CONCLUSIONES}

La función tamaño - densidad desarrollada utiliza la ecuación propuesta por Jack y Long (1996) que considera el logaritmo natural del diámetro medio cuadrático como la variable del tamaño medio en función del logaritmo natural de la densidad. Los parámetros obtenidos en el ajuste de la función son similares a los resultados obtenidos por Venegas (1999) para la misma especie y no comparable al estudio de Donoso et al. (2007), ya que estos últimos autores utilizaron el formato propuesto por Reineke (1933).

La obtención de la línea de la máxima relación tamaño densidad mediante la corrección gráfica del parámetro interceptor $\left(b_{1}\right)$, a partir de la función de tamaño densidad ajustada mediante mínimos cuadrados ordinarios, es un método ampliamente utilizado por ser simple y arrojar estimaciones robustas. Sin embargo, Zhang et al. (2005) plantean que el método de los mínimos cuadrados ordinarios puede producir líneas de autorraleo con pendientes inadecuadas, debido a que este método es sensible a los datos seleccionados para el ajuste; no obstante, su utilidad ha quedado demostrada por diversos estudios realizados a nivel mundial.

La curva de la relación de tamaño - densidad ajustada, representa el promedio de las situaciones de los rodales, aun cuando esta relación teóricamente es independiente de las condiciones de sitio (Drew y flewelling 1979, Newton 1997, Saunders y Puettmann 2000, Shaw y Long 2007). Al respecto, Donoso et al. (2007) probó que para $D$. winteri las condiciones fisiográficas y composición de especies afectan solo parcialmente estas relaciones, sugiriendo que fundamentalmente los cambios que ocurren según variaciones de sitio se relacionan con diferencias en las especies acompañantes en estos bosques secundarios dominados por $D$. winteri. Este es un tema en el cual se debería profundizar debido a las implicancias potenciales al momento de evaluar esquemas de manejo y controlar el crecimiento de los rodales. Esto sugiere ampliar la capacidad del diagrama mediante la introducción de los ejes adicionales, como la composición de especies y posiblemente calidad de sitio, como lo plantea Farnden (2002).

La utilidad de incorporar las isolíneas de altura y volumen permite tener una estimación del estado cuantitativo del rodal. La isolínea de la altura permite realizar una mejor proyección de las intervenciones propuestas, al combinar los diagramas con funciones de altura dominante/edad es factible estimar la edad de los rodales y por lo tanto el tiempo que tomará llegar a un estado de desarrollo para los esquemas de manejo proyectados, tal y como lo plantean Vacchiano et al. (2008) y Castedo et al. (2009). Además durante el ejercicio de proyección de los rodales, permiten diferenciar tipos de raleos, si la trayectoria del raleo se traza paralela a las isolíneas de altura, manteniendo la altura media dominante constante, simula un raleo por lo bajo ya que en esta intervención se eliminan aquellas clases de copas que están suprimidas, moribundas o cuya posición es intermedia a baja (Daniels et al. 1982).

En relación a las líneas de densidad relativa definidas para renovales de $D$. winteri de $0,55,0,40,0,30$ y de 0,15 , en base al análisis de mortalidad, desarrollo de copas de las parcelas del presente estudio y a los trabajos de Navarro et al. (1999) y Venegas (1999), es posible sugerir las siguientes densidades relativas para efectuar manejo en renovales de $D$. winteri con objetivos productivos maderables:

- Mantener densidades relativas sobre un 0,55 no es recomendable ya que en esta zona se produce una alta mortalidad por competencia, la tasa de crecimiento del rodal es mínima, los rodales que se manejen sobre esta densidad relativa demorarán más años en alcanzar el bosque meta. Esto lo ilustra la parcela testigo con cifras de 0,50 a 0,78 de densidad relativa y alta mortalidad. En esta zona cualquier incremento del árbol medio irá acompañado de mortalidad (Drew y Flewelling 1979, Newton 1997, Saunders y Puettmann 2000, Shaw y Long 2007). Al respecto, Nyland (2002) indica que densidades relativas hasta un 0,80 podrían justificarse, ya que en estos casos la mortalidad se concentra en individuos suprimidos o intermedios. Sin embargo, para los renovales estudiados de $D$. winteri que presentan índice de densidad relativa sobre 0,55 , sus tasas de crecimiento en diámetro son mínimas, lo que prolonga el tiempo de cosecha de los árboles futuros de manera importante.

- Con densidades de manejo entre 0,40 a 0,55 de índice de densidad relativa se logra maximizar la producción de biomasa para uso energético o tableros. De acuerdo a lo experimentado, los rodales que se ubiquen en esta zona presentan menor riesgo de caída por viento.

- Manejar renovales dejando densidades relativa entre un 0,15 a 0,40 permite concentrar el manejo para madera de alta calidad. Sin embargo, para reducir el riesgo de daño por viento y permitir ingresos en periodos más cortos, lo que es especialmente importante en economías de pequeños y medianos productores forestales, es recomendable densidades relativas límites de 0,30 y 0,45 . El tratamiento de raleo moderado representa esta condición, que significa realizar intervenciones cada 8 a 15 años, aumentando su periodicidad en los raleos posteriores con intensidades de extracción que varían de $24 \%$ de índice de densidad relativa en el primer raleo y $15 \%$ en los raleos sucesivos

- Ubicar rodales en densidades relativas inferiores a 0,15 significa una subutilización del sitio y máximos crecimientos a nivel de árbol. Al respecto, Gezan et al. (2007) plantean que densidades bajo esta línea podrían indicar que se requiere enriquecer el rodal. Esta situa- 
ción queda representada por el raleo severo, donde se redujo la densidad relativa de 0,50 a 0,12 , quedando en la zona de crecimiento libre el rodal durante diez años. A nivel de árboles individuales esto significó un aumento del área de copa de los árboles dominantes 2,6 veces respecto del testigo y 1,8 veces el tamaño del árbol de área basal media; a nivel de rodal la producción física disminuyó en un $60 \%$ respecto del testigo.

El diagrama desarrollado, permite evaluar regímenes de raleos tardíos orientados a la producción de madera de mayor precio en Chile. El uso del diámetro medio cuadrático en la ordenada y la densidad en la abscisa permite a los silvicultores y potenciales usuarios visualizar con mayor facilidad la relación entre la densidad relativa y el tamaño medio de los árboles a través del diámetro medio cuadrático en vez del volumen medio, tal y como lo plantean Farnden (2002) y Long y Shaw (2005).

Al momento de utilizar el diagrama en un rodal de D. winteri, los usuarios deben considerar sus limitaciones:

- Las líneas de densidad relativa son sensibles a la composición de especies del rodal (Donoso et al. 2007). En este caso, la composición de especies fue restringida a rodales con dominancia de $D$. winteri, con al menos un 70 \% del área basal de esta especie y no se analizó en términos específicos las especies acompañantes y su variabilidad.

- La dinámica y los regímenes de manejo descritos para la situación promedio que representa el diagrama no son necesariamente verdaderos u óptimos para cualquier rodal en particular, pero sí útiles. Es recomendable en consecuencia considerar funciones de índice de sitio para lograr predicciones de desarrollo del rodal más exactas.

- Los diagramas de manejo de la densidad no incorporan la variable calidad del árbol, sin embargo, en renovales de canelo dada sus altas densidades y la aptitud genética de la especie de formar fustes bastantes rectos, es admisible usar estos diagramas como herramienta de manejo.

Es factible desarrollar un diagrama de manejo de la densidad de segundo nivel de complejidad para renovales de $D$. winteri, fundamentado en los marcos teóricos del principio de autorraleo y la experiencia de su elaboración en otras especies a nivel mundial, con errores aceptables para la planificación y control de tecnologías silvícolas. La planificación de raleos tardíos en renovales de $D$. winteri, en sitios de productividad media, utilizando el diagrama de manejo de la densidad y basado en la experiencia de ensayos de raleos y manejos operacionales, hacen recomendable mantener niveles de ocupación de sitio entre las zonas de subutilización (índice de densidad relativa de 30 $\%$ y la de inminente mortalidad por competencia (índice de densidad relativa de $45 \%$ ), realizando al menos tres raleos para alcanzar un rodal meta que permita aportar productos aserrables y debobinables a una edad no menor a los 80 años para el sitio analizado. Esto reduce el riesgo de caída de árboles por viento y la pérdida de producción física del sitio.

\section{AGRADECIMIENTOS}

Los autores expresan su agradecimiento al Sr. Julio Pinares, por su colaboración y apoyo logístico con el estudio.

\section{REFERENCIAS}

Avery TE, HE Burkhart. 1994. Forest Measurements. Nueva York, USA. McGraw-Hill. 408 p.

Castedo F, F Creciente, P Álvarez, M Barrio. 2009. Development of a stand density Management diagram for radiata pine stand including assessment of stand stability. Oxford Journal Life Sciences Forestry 82 (1): 1-16.

Curtis R. 1970. Stand density measures: An interpretation. Forest Science 16:403-414.

Di Castri F, ER Hajek. 1976. Bioclimatología de Chile. Santiago, Chile. Editorial de la Universidad Católica de Chile. 129 p.

Donoso C, B Escobar, M Cortes, P Donoso. 1985. Regeneración en bosques de la Cordillera de la Costa V Parte. Valdivia, Chile. Facultad de Ciencias Forestales, Universidad Austral de Chile (Informe de Convenio $N^{\circ}$ 86). 216 p.

Donoso PJ, D Soto, RA Bertín. 2007. Size- density reationships in Drimys winteri secondary forest of the Chiloe Island, Chile: effects of fisiografy and species composition. Forest Ecology and Management 239: 120-127.

Drew J, J Flewelling. 1977. Some recent Japanese theories of yield-density relationships and their application to monterey pine plantations. Forest Science 23: 517-534.

Drew J, J Flewelling. 1979. Stand density management: a alternative approach and its application to Douglas-fir plantations. Forest Science 25(3): 518-532.

Farnden C. 2002. Recommendations for constructing stand density management diagrams for the Province of Alberta. Report to Alberta Land and Forest Division. Alberta, Canada. Ministry of Sustainable Resource Development. 17 p.

Gezan S, A Ortega, E Andenmatten. 2007. Diagramas de manejo de la densidad para renovales de roble, raulí y coigüe en Chile. Bosque 28(2): 97-105.

Gujarati D. 2003. Econometría. México, D.F. Mcgraw-hill 4ª edición. 1028 p.

INFOR (Instituto Forestal, CL). 2004. Estudio de mercado para madera de canelo en Estados Unidos y Europa. 92 p. (Informe técnico $\mathrm{N}^{\circ} 167$ ).

Jack S, J Long. 1996. Linkages between silviculture and ecology: An analysis of density managenment diagrams. Forest Ecology and Management 86: 205-220.

Long, JN, JD Shaw .2005. A density management diagram for even-aged ponderosa pine stands. West. Journal of Applied Forestry 20(4): 205-215.

Navarro CO, C Donoso, V Sandoval. 1999. Los renovales de Canelo. In Donoso C, A Lara eds. Silvicultura de los bosques nativos de Chile. Santiago, Chile. Editorial Universitaria. p. 341-380.

Newton P. 1997. Stand density management diagrams: Review 
of their development and utility in stand-level management planning. Forest Ecology and Management 98: 251- 265.

Nyland R. 2002. Silviculture. Concepts and Applications. New York, USA. McGraw-Hill Companies. 633 p.

Penner M, E Swift, R Gagnon, R Brissette. 2006. A stand density management diagram for balsam fir in New Brunswick. Forestry Chronicle 82(5): 700-711.

Pretzsch H. 2009. Forest Dynamics, Growth and Yields: From Measurements to Model. Berlin, Germany. Springer-Verlag Berlin Heidelberg. 695 p.

Reineke L. 1933. Perfecting a stand density index for even-age forest. Journal of Agricultural Research 46: 627-638.

Reyes R, Donoso P, Donoso C, Navarro C. 2009. Crecimiento de renovales de Drimys winteri después de 16 años de aplicados distintos tratamientos de raleo en las cordilleras de Los Andes y de la Costa en Chile. Bosque 30(3): 117-126.

Saunders M, K Puettmann. 2000. A preliminary White Spruce density management diagram for the lake states. Minnesota, USA. Departament of forest resources, University of Minnesota (Staff papers series $N^{0} 145$ ). $21 \mathrm{p}$.

Shaw J, J Long. 2007. Density management diagram for Longleaf Pine stands with aplication to Red-Cockaded Woodpecker habitat. South. Journal of Applied Forestry 31(1): 28-38.

Smith N, D Hann . 1984. A new analitical model based on the $-3 / 2$ power rule of self-thinning. Journal of Forest Re- search 14: 605-609.

Somers G, R Farrar. 1991. Biomatematical growth equations for natural longleaf pine stands. Forest Science 37(1): 227-244.

Solomon D, L Zhang. 2002. Maximun size-density relationships for mixed softwoods in the northeastern USA. Forest Ecology and Management 155: 163-170.

Vacchiano G, R Motta, J Long, J Shaw. 2008. A density management diagram for Scots pine (Pinus sylvestris L.): a tool for assessing the forest's protective effect. Forest Ecology and Management 255(7): 2542-2554.

Valbuena P, C Peso, F Bravo. 2008. Stand density Management diagrams for two mediterranean pine species in Eastern Spain. Investigación Agraria Sistemas y Recursos Forestales 17(2): 97-104.

Venegas J. 1999. Estudio de La Ley de -3/2 c de Auto-Raleo en Renovales de Drimys winteri Forst. en la X Región”. Tesis Ingeniería Forestal. Temuco, Chile. Facultad de Recursos Naturales, Universidad Católica de Temuco. 65 p.

Yoda K, T Kira, H Ogawa, K Hozumi. 1963. Self-thinning in overcrowded pure stands under cultivated and natural conditions (Intraspecific competition among higher plants XI). Journal of Biology 14: 107-129.

Zhang L, H Bi, J Gove, L Heath. 2005. A comparasion of alternative methods for estimating the self-thinning boundary line. Canadian Journal of Forest Research 35: 1507-1514.

Recibido: 18.01 .10

Aceptado: 20.05.11 